\title{
Prevención del embarazo a temprana edad a través de la cátedra de educación sexual como estrategia pedagógica apoyada en $\mathrm{TIC}^{1}$
}

\section{Prevention of the pregnancy to early age across the chair of sexual education like pedagogic strategy}

DOI: http://dx.doi.org/10.17981/cultedusoc.9.3.2018.100

Artículo de investigación. Fecha de recepción: 15/06/2018. Fecha de aceptación: 27/11/2018

\author{
John Jairo Lara Martinez²; \\ Yosiris Ospino, Yenis Tamara; Angelica Andrade; \\ Joel Ospino; Elmira Salas; Glenis Rivera y Yomaris Bertel ${ }^{3}$ \\ I.E.D Técnica Agropecuaria Pestalozzi (Colombia) \\ laramjo@live.com
}

Para citar este artículo:

Lara, J., Ospino, Y., Tamara, Y., Andrade, A., Ospino, J., Salas, E., Rivera, G. y Bertel, Y. (2018). Prevención del embarazo a temprana edad a través de la cátedra de educación sexual como estrategia pedagógica apoyada en TIC. Cultura. Educación y Sociedad, 9(3), 841-848. DOI: http://dx.doi.org/10.17981/cultedusoc.9.3.2018.100

\section{Resumen}

El presente articulo tiene como objetivo prevenir los embarazos adolescentes a partir de una cátedra de educacion sexual con estudiantes de bachillerato debido a la alta deserción que se presenta en la institución. La metodologia del estudio se desarrollo desde un enfoque cualitativo con un alcance descriptivo utilizando como tecnicas de recolección de informacion la obervacion participante e intrumentos como la encuesta, la poblacion participante corresponde a sesenta (60) estudiantes de la I.E.D Técnica Agropecuaria Pestalozzi. Los resultados que se alcanzaron con la aplicación del proyecto fue que las estudiantes lograron identificar en que temas tenían conocimiento y cuanto, además especificaron que contenidos deben profundizarse y tratarse en el blog como medio de socialización.

Palabras claves: educacion sexual, adolescencia, embarazo adolescente.

\section{Abstract}

The present article has as aim anticipate the teen pregnancies from a chair of sexual education with students of baccalaureate due to the high desertion that one presents in the institution. The methodology of the study I develop from a qualitative approach with a descriptive scope using as technologies of compilation of information the obervacion participant and intrumentos as the survey, the population participant corresponds to sixty (60) students of the Technical Agricultural I.E.D Pestalozzi. The results that were reached by the application of the project it was that the students managed to identify in that topics had knowledge and all that, in addition they specified that contained they must be deepened and to treat itself in the blog as way of socialization.

Keywords: sexual education, adolescence, teen pregnancy.

1 Este artículo ha sido derivado del Programa de Fortalecimiento de la Cultura Ciudadana y Democrática CT+I a través de la IEP apoyada en TIC en el Departamento de Magdalena: CICLON

2 Líder del Grupo de Investigación "Pirsag".

3 Docentes miembros del Grupo de Investigación Pirsag, de la I.E.D Técnica Agropecuaria Pestalozzi.

- The author; licensee Universidad de la Costa - CUC.

Cultura, Educación y Sociedad vol. 9 no. 3, pp. 841-848. Diciembre, 2018

Barranquilla. ISSN 2389-7724 Online 


\section{Introducción}

Actualmente existen diversas problemáticas alrededor de la población adolescente, entre estas encontramos el consumo de sustancias alucinógenas, consumo de alcohol, deserción escolar y embarazos a temprana edad. La adolescencia como etapa del desarrollo es concebida como un periodo donde hay muchas dudas, se establece el proyecto de vida, metas y a nivel emocional se constituyen vínculos sólidos pero a la misma vez se pretende alcanzar la independencia como ser humano, acompañándose de cambios corporales, familiares y sociales (Quintero \& Rojas, 2015).

Por lo anterior el grupo de investigación decide realizar un estudio donde se lleve a cabo la prevención de embarazos adolescentes a través de la implementación de una cátedra de educación sexual, donde los estudiantes puedan identificar cuáles son los aspectos más relevantes en cuanto a la vida sexual activa y que grado de responsabilidad deben asumir, esta iniciativa parte de la alta deserción que vivencia en la institución.

\section{Adolescencia}

La Organización Mundial de la Salud (OMS) como ente rector en salud determina los rangos de edad para el periodo de la adolescencia, estos responden a una subdivisión donde se estipula que los jóvenes de 10-13 años están en una adolescencia temprana, 14-16 años en una adolescencia media y 17-20 años una adolescencia tardía (Organizacion Mundial de la Salud, 2018).

En este sentido también se define la adolescencia como una etapa de desarrollo en la que el sujeto sufre una transición de gran importancia teniendo como características particulares el crecimiento acelerado y cambios de distintas índoles (corporales y emocionales) que se encuentran supeditados a procesos biológicos, contem- plándose que el inicio de esta es la pubertad (Mañeru, 1999).

Por lo anterior se menciona que en la adolescencia aparecen los retos personales, es el momento donde los jóvenes aprenden de sus experiencias y logran puntos significativos en su desarrollo cognitivo que le permiten alcanzar niveles de madurez e independencia en ámbitos como el psicológico, emocional y físico, adquiriendo herramientas en su formación que le permitirán desenvolverse de manera satisfactoria o negativa en su vida adulta dependiendo de su fortalecimiento (Contreras, 2011).

\section{El embarazo adolescente}

El inicio de una vida sexual activa a temprana a edad enmarca un riesgo latente en cuanto a que una adolescente presente un embarazo precoz que podría generarse en su mayoría por falta de orientación, comunicación efectiva y acompañamiento por parte de sus cuidadores en el proceso de maduración mental y físico (Quintero \& Rojas, 2015). Por esta razón el embarazo adolescente se conceptualiza como evento que sucede en los dos (2) primeros años de edad ginecológica; es decir después de la primera menstruación (menarquia), o cuando la joven se encuentra aún bajo el cuidado y manutención de sus padres (Montenegro \& Pacheco, 2010).

Asimismo se establece que los embarazos adolescentes son más recurrentes en comunidades que tienen un alto índice de pobreza, rurales o bajos niveles de educación; además se estima que en la mayoría de los casos las jóvenes no saben cómo evitar el embarazo, no conocen como solicitar métodos anticonceptivos, como usarlos o simplemente siente pena ante la idea de comprarlos y no tienen el dinero para ello (Organizacion Mundial de la Salud, 2018).

Es importante mencionar que el tema de la sexualidad es amplio y en los adolescentes es de vital importancia indicarles que abarca más aspectos que solo el desarrollo 
genital, estos corresponden a las relaciones con el sexo opuesto, al desarrollo anatómico, a las condiciones sociales y culturales. En este orden de ideas también contiene que conozcan su intimidad, cuales son los roles que asumirán en una relación de pareja, que comprendan sus cambios corporales, que se adapten al erotismo que hace presencia como nuevo elemento de vida $\mathrm{y}$ entender como son los procesos reproductivos (acciones y consecuencias) (Muñoz, Jiménez \& Moreno, 2008).

\section{Educación sexual}

Se considera el periodo de la adolescencia como una etapa de transición a la edad adulta donde con frecuencia se asumen riesgos por el hecho de poseer fisiológicamente condiciones óptimas, además el ámbito sexual y reproductivo toman importancia por lo que la educación en estos campos llega a ser piedra angular en una formación integral referentes a las competencias del ser (Cacéres, Castro, Sarmiento, \& Rodriguez, 2003).

En este orden la Ley General de Educación en su Art 14, estipula de manera obligatoria implementar en las Instituciones Educativas la educación sexual para complementar necesidades psíquicas, físicas y emocionales de los estudiantes dependiendo su edad (Ministerio de Educacion , 1994). De acuerdo a lo anterior la educación sexual tiene por objetivo mejorar la calidad de vida de los estudiantes a partir de brindar estrategias que le permitan un desarrollo integral y prevenir frustraciones en cuanto a las aspiraciones profesionales y laborales, y que además en el Proyecto Educativo Institucional se contengan las políticas necesarias para cumplir las metas trazadas en esta temática (Cacéres, Castro, Sarmiento, \& Rodriguez, 2003).

En este sentido la educación sexual se relaciona con los embarazos adolescentes en la medida que; en termino de porcentajes en un $36 \%$ no cuentan con educación sexual, el $32 \%$ solo han cursado básica primaria, $21 \%$ no tienen educación formal y el $28 \%$ asiste a un centro educativo; por lo tanto se denota que el nivel de educación incide de manera directa en la comprensión de la responsabilidad con la que se asume la sexualidad en la adolescencia (Cacéres, Castro, Sarmiento, \& Rodriguez, 2003).

\section{Metodología}

La investigación fue desarrollada desde un enfoque cualitativo con un alcance descriptivo con un diseño de investigación- acción, teniendo en cuenta que los docentes pretenden prevenir el embarazo adolescente en las estudiantes de bachillerato observando y caracterizando en primera instancia la problemática y posteriormente interviniendo con las actividades que se diseñen en el procedimiento, teniendo en cuenta que la teoría plantea que se identificarán los puntos más significativos de la población participante de la investigación (Hernandez Sampieri, 2014).

\section{Participantes}

Las estudiantes participantes del estudio son sesenta (60) niñas de los grados $9^{\circ}, 10^{\circ}$ y $11^{\circ}$ entre edades de 14 a 16 años, de la Institución Educativa Técnica Agropecuaria Pestalozzi en el municipio de Nueva Granada, Magdalena.

\section{Técnicas e instrumentos} de recolección de datos

Los investigadores establecieron como técnica de recolección la observación directa y como instrumentos el diario de campo como medio de registro de la información y la encuesta para obtener de manera particular la información de cada sujeto participante, permitiendo a su vez determinar cuáles son los temas menos conocidos por las estudiantes y cuales es la causa por la cual se dan ciertos comportamientos. 


\section{Procedimiento}

El desarrollo de la investigación se dio de manera que los investigadores realizaran la observación directa durante toda la aplicación de las actividades, determinando aspectos claves del comportamiento, actitudes y nivel de conocimiento de las estudiantes mediante una mesa redonda. Asimismo se diseñó una encuesta que contiene catorce (14) preguntas referentes a la temática trabajada que es Educación Sexual en función de la prevención del embarazo adolescente posterior a ello se ejecutaran talleres de capacitación frente a estos contenidos y se hará una socialización de los resultados y de los temas más relevantes a la comunidad estudiantil en general a través de la creación de un blog.

\section{Resultados}

A continuación se expondrán los hallazgos encontrados durante la aplicación de las actividades diseñadas, en cuanto a la observación inicial realizada por los maestros a las 60 estudiantes que fueron reunidas en un salón de audiovisuales de la institución donde se organizaron en mesa redonda para conocer que concepciones tenían sobre el embarazo adolescente, si conocían su proceso y consecuencias tanto físicas, como psicológicas y emocionales; ante esto las estudiantes contestaron lo siguiente. (Ver Tabla 1)

TABLA 1

Mesa Redonda

\begin{tabular}{|c|c|c|c|}
\hline Sesión & Actividad & $\begin{array}{l}\text { Descripción de } \\
\text { actividad }\end{array}$ & Resultados \\
\hline Sesión 1 & Mesa redonda & $\begin{array}{l}\text { En la presente actividad } \\
\text { las estudiantes fueron } \\
\text { citadas en el audiovisual } \\
\text { o de tecnologías de la } \\
\text { institución. En este } \\
\text { espacio se organizaron a } \\
\text { manera de mesa redonda } \\
\text { para desarrollar el panel } \\
\text { en el que los maestros } \\
\text { conocerán que nivel de } \\
\text { conocimiento acerca del } \\
\text { embarazo adolescente, } \\
\text { cuales es su proceso y } \\
\text { qué consecuencias tiene. }\end{array}$ & $\begin{array}{l}\text { En primera instancia los maestros observaron } \\
\text { la actitud de las estudiantes, estas manifestaron } \\
\text { interés e inquietud frente a la temática, y además } \\
\text { curiosidad debido a que en el grupo se encontraban } \\
\text { dos jovencitas que son madres adolescentes. } \\
\text { En este sentido se identificó que el nivel de } \\
\text { conocimiento de las estudiantes es bastante } \\
\text { amplio, por la razón de que las experiencias de } \\
\text { las compañeras le permiten conocer el proceso } \\
\text { y que implicaciones físicas tiene; por ejemplo } \\
\text { mencionaron, "en el embarazo adolescente las } \\
\text { muchachas casi no pueden salir porque deben } \\
\text { estar en reposo, tienen que estar pendiente que no } \\
\text { se vayan a caer, que tienen que comer a tiempo } \\
\text { y comidas sanas", "sé que hay niñas que se han } \\
\text { muerto porque no tienen el cuerpo preparado } \\
\text { para el embarazo, porque no se le ha madurado } \\
\text { por completo su cuerpo", "yo sé que al cumplirse } \\
\text { los } 9 \text { meses de embarazo pues las adolescentes } \\
\text { pasan por el parto ya sea natural o por cesárea, } \\
\text { y algunas se ven grave porque no tienen el cuerpo } \\
\text { preparado". }\end{array}$ \\
\hline
\end{tabular}

Fuente: Elaboración propia. 
La encuesta realizada a las estudiantes pretendió indagar el nivel de conocimientos acerca de educación sexual, cuales son los temas más manejados de manera adecuada y responsable por los estudiantes y determinar cuáles son las temáticas a trabajar en el blog como forma de socialización. Los resultados encontrados fueron qué; con relación a la pregunta $A$, las estudiantes contestaron que las personas más indicadas para hablar de estos temas son los padres, referente a la pregunta $B$, las estudiantes en su mayoría contestaron que de la edad ideal es de 12-14 años y un pequeño porcentaje puntuó la opción de nunca argumentando que es lo único que le evitaría un embarazo.

En cuanto a la pregunta $C$, las estudiantes contestaron que los métodos que conocen para evitar un embarazo son los anticonceptivos, la abstención y el coito interrumpido, la pregunta $D$, se comparten las opiniones en la medida que la mitad de la población encuestada cree que cuando se tratan estas temáticas los estudiantes Algunos no comentan por vergüenza al tema y el otro porcentaje considera que Toman el tema con seriedad e importancia, porque no quieren tener un embarazo y continuar con su proyecto de vida. En la pregunta $E$, las estudiantes respondieron en su totalidad que conocen lo suficiente de sexo y en la pregunta $F$ expresan en su mayoría que la palabra condón alude a prevención de embarazos.

Frente a la pregunta $G$ las estudiantes anotan que a veces hablan de sexualidad, en la pregunta $H$, manifestaron que cuando necesitan orientación en contenidos de sexualidad generalmente acuden a los amigos. En la pregunta $I$ la mitad del grupo apunto a que uno de los motivos por los que no tienen relaciones sexuales es por temor a sus padres y la otra mitad por evitar un embarazo no deseado, la siguiente pregunta $(J)$, las estudiantes contestaron en un alto porcentaje si a que si han mantenido relaciones sexuales, mientras que el porcentaje restante respondió que no, entonces en la pregunta siguiente que podía ser o no respondida, el porcentaje que dijo no haber tenido relaciones sexuales no colocaron edad, y las que contestaron que si algunas colocaron la edad que oscilo entre 15-16 años, y otras se abstuvieron de colocar el dato.

En la siguiente pregunta $L$, las estudiantes enunciaron que si hablaban de su sexualidad y que lo hacían con sus amigos $y$ padres, la pregunta $M$ refiere a que concepción tienen los padres de las relaciones sexuales y las estudiantes coincidieron en que ellos piensan que pueden tenerlas pero que deben cuidarse de manera responsable.

En relación a la pregunta $N$, respondieron las estudiantes que si han recibido información formal sobre educación sexual precisamente en la institución donde estudian, la pregunta $O$ refiere a los temas de sexualidad que más manejan y las estudiantes manifestaron que son; métodos anticonceptivos, menarquia, partes y cambios del cuerpo y la pregunta siguiente $(P)$ las estudiantes contestaron que les gustaría profundizar en temas como enfermedades de transmisión sexual, el embarazo y relaciones sexuales, finalmente estas propusieron que los temas que se incluyeran en el blog fueran conocimiento de las partes íntimas, proceso de concepción y el embarazo.

Posterior a esto se realizaron talleres con las temáticas de interés de las estudiantes plasmadas en la encuesta durante estos se mostraron participativas y receptivas a lo impartido por los docentes, al final de cada sesión se realizaba un sondeo para establecer en qué medida se iban esclareciendo inquietudes y fortaleciendo el conocimiento ya existente. Finalmente se publicaron los contenidos sugeridos en el blog denominado PIRSAG (pirsagpestalozzi.simplesite.com) entre estos estuvieron, Educación sexual en los niños por medio de capítulos de cortometrajes, estos tenían temas de las partes genitales de hombres y mujeres, proceso de concepción y exposición de los resultados del proyecto por parte de los investigadores. 


\section{Discusión}

La aplicación de la investigación por parte de los docentes en cuanto a la prevención del embarazo adolescente a través de la catedra de Educación Sexual de la institución permitió determinar que las adolescentes conocen ciertos temas de sexualidad de manera amplia, pero no contemplan la responsabilidad necesaria para desenvolverse en estos.

En cuanto al tema del embarazo adolescente estas conocen las repercusiones físicas, pero no tienen una idea de las afectaciones emocionales y que no solo la sexualidad se trata de mantener relaciones sexuales sino también abarca otros conceptos como, cambios corporales, protección y tipo de relaciones de pareja; así como lo plantea Muñoz, Jiménez \& Moreno (2008), la sexualidad es un tema extenso de gran relevancia donde los niños y adolescentes deben comprender que no solo hay un desarrollo sexual sino que también se contemplan relaciones afectivas y cumplir con estatutos sociales y culturales.

Además el blog, los talleres y la percepción del nivel de conocimiento sobre se- xualidad que poseen las estudiantes los maestros lo implementaron como herramientas que le dan paso a promover una educación sexual entre los adolescentes y profundizar en otros temas que de manera implícita inciden en el desarrollo de esta temática estos pueden ser; el conocimiento del cuerpo, la comunicación con los padres y la autoestima, ya que en la adolescencia se definen una serie de aspectos enfocados hacia la construcción de su proyecto de vida y constitución de vínculos emocionales que le van a brindar firmeza en la transición de los cambios que se experimentan en esta etapa (Quintero \& Rojas, 2015).

Por último los maestros investigadores sugieren que se realicen jornadas en las que se involucre a toda la comunidad en general, en estas se lleven a cabo talleres estudiantiles y capacitaciones donde participen maestros y padres de familia con el fin de fortalecer las estrategias pedagógicas en educación sexual, pero apuntando también a otros contenidos que hacen parte del desarrollo infantil y juvenil, como por ejemplo el establecimiento de metas personales y que esto a su vez aporta a disminuir los índices de deserción en la escuela.

\section{Anexos}

\section{"Encuesta sobre educación sexual"}

\section{- Instrucciones:}

- Lee con atención cada pregunta de la encuesta.

- Marca con una X la alternativa que consideres cercana a tu realidad.

- Si la pregunta lo requiere puedes marcar más de una alternativa.

a) Consideras que la persona adecuada para conversar sobre sexualidad es: Padre Madre Ambos Profesores_____Amigos_____Otros (especificar)

b) Qué edad consideras adecuada para tratar estos temas por primera vez?

De 5 a 8 de 9 a 11 de 12 a 14 Nunca 
c) Conoces formas efectivas de evitar un embarazo? Si es así, menciona tres:

a)

b)

c)

d) La reacción de los estudiantes de tu clase al tratar estos temas en el colegio es:

Toman el tema con seriedad e importancia

No toman el tema con seriedad e importancia

Algunos no comentan por vergüenza al tema

No participan y no le dan seriedad al tema

No se toca el tema en el colegio

e) ¿Cuánto crees tú qué sabes sobre "sexo"?

mucho ____poco ____lo suficiente

f) La palabra condón te hace pensar en:

Prevención de Enfermedades de Transmisión Sexual (ETS)

Prevención de Embarazos

Vergüenza

Otros (especificar)

g) ¿Con qué frecuencia hablas sobre "sexualidad"?

Siempre___ A veces___Mu poco__- Nunca

h) Si necesitas orientación sobre estos temas, hablas con: amigos ____padres especialistas nadie profesores

i) ¿Por cuál de los siguientes motivos no tendrías relaciones sexuales? ETS

Embarazos no deseados

Mis padres

Vergüenza

No me siento listo(a)

Otros (especificar)

j) ¿Has tenido relaciones sexuales?

Sí_-___ No

k) ¿A qué edad tuviste o tendrías tu primera relación sexual? (PREGUNTA OPCIONAL)

l) ¿Has hablado con alguien sobre tu sexualidad?

Sí_-_ No

Con quién?

Familiares Amigos____-_ Otros

m) ¿Qué opinan tus padres sobre las relaciones sexuales entre los adolescentes? Que deben esperan hasta el matrimonio Que pueden tenerlas si se cuidan responsablemente

-.--- Rechazan absolutamente el tema y se molestan al hablar de ello

No les interesa

No hablamos del tema

n) ¿Has recibido formalmente información sobre educación sexual?

Sí_____ No

o) ¿Qué temas son los que más manejas sobre la sexualidad?

p) ¿Qué temas te gustaría manejar más?

q) Para ayudarnos, anota conceptos o ideas que serían necesarias incluir en un tríptico informativo sobre sexualidad: 


\section{Referencias}

Contreras Britto, J. B. (2011). Prevalencia de embarazo en adolescentes en la E.S.E Alejandro Prospero de Santa Marta en los años 2008- 2009. Bogotá: Universidad Nacional de Colombia.

Cacéres, M., Castro, L., Sarmiento, N., \& Rodriguez, A. (2003). Educacion sexual y los adolescentes del municipio de Arauca. Arauca : Universidad Nacional de Colombia.

Mañeru, A. (1999). Autoridad femenina en educación. Kikiriki, 54, 26-31. Mañeru, Ana (2007). La práctica de la autoridad en las relaciones. Crítica, 943, 55-59.

Ministerio de Educacion . (08 de 02 de 1994). Ley 115 de Febrero 8 de 1994. Obtenido de https://www.mineducacion. gov.co/1621/articles-85906_archivo_ pdf.pdf

Montenegro, D. \& Pacheco, C. (Abril de 2010). Narrativas de adolescentes embarazadas populares que han planificado su embarazo. Trabajo para optar al grado de Magíster en Psicología Clínica. Santiago de Chile.

Muñoz T; Jiménez I; y Moreno, M. (2008). Reputación conductual y género en la adolescencia. Anales de psicología, 24(2), 334-340. Disponible en: http://redalyc.uaemex.mx/pdf/167/16711589017. pdf

Organizacion Mundial de la Salud . (23 de 02 de 2018). El embarazo en la adolescencia . Obtenido de http://www.who.int/ es/news-room/fact-sheets/detail/adolescent-pregnancy

Quintero, A., \& Rojas, H. (2015). El embarazo a temprana edad, un analisis desdela perspectiva de madresadolescentes. Revista virtual Universidad Catolica del Norte , 222-237.

UNESCO (2010). Orientaciones Técnicas internacionales sobre Educación de la Sexualidad. https://es.unesco.org/

UNESCO (2012). Los jóvenes y las competencias: trabajar con la educación. Informe de seguimiento de la educación para todos en el mundo. https://es.unesco. org/ 